Check for updates

Cite this: RSC Adv., 2017, 7, 44035

Received 6th June 2017

Accepted 7th September 2017

DOI: $10.1039 / c 7 r a 06306 g$

rsc.li/rsc-advances

\section{A dual-faced interlayer prepared by electron beam evaporation for enhanced-performance lithium- sulfur batteries}

\author{
Qiong Tang, (DD ab Heqin Li, ${ }^{\text {a }}$ Jing Zhang, ${ }^{\text {ab }}$ Zhiwei Lin, ${ }^{a}$ Yuanyuan Pan, ${ }^{\text {a }}$ \\ Qingzhuo $\mathrm{Hu}^{\mathrm{a}}{ }^{\mathrm{Yu}} \mathrm{You}^{\mathrm{b}}$ and Yangwei Ye ${ }^{\mathrm{b}}$
}

In this work, a dual-faced carbon paper was prepared by depositing $\mathrm{Al}_{2} \mathrm{O}_{3}$ on one side of carbonized filter paper via the technique of electron beam evaporation. Assembled into $\mathrm{Li}-\mathrm{S}$ batteries, the $\mathrm{Al}_{2} \mathrm{O}_{3}$-deposited carbon paper served as a multifunctional interlayer. The cathode of $70 \%$ sulfur content with this interlayer presented notable enhancements in electrochemical performance in contrast to that with a single carbon interlayer. At a current rate of $0.5 \mathrm{C}$, the battery with the $\mathrm{Al}_{2} \mathrm{O}_{3}$-deposited carbon interlayer delivered a high initial capacity of $1253 \mathrm{~mA} \mathrm{~h} \mathrm{~g}^{-1}$ and retention of $700 \mathrm{~mA} \mathrm{~h} \mathrm{~g}{ }^{-1}$ over 120 cycles, while the battery with the carbon interlayer delivered an approximate initial capacity of $1117 \mathrm{~mA} \mathrm{~h} \mathrm{~g}^{-1}$ but much poorer retention of $441 \mathrm{~mA} \mathrm{~h} \mathrm{~g}^{-1}$ over 87 cycles. The carbon side of the interlayer was placed toward the cathode as the upper current collector, which ensured the conductivity of the contact interface with the active material and promoted activation of sulfur. At the same, $\mathrm{Al}_{2} \mathrm{O}_{3}$ as a polar material facing toward the separator impeded the leakage of soluble polysulfides and mitigated the shuttle effect by chemical adsorption of soluble polysulfides. Combined with the interstitial structure of the interlayer acting as a physical container, Li-S batteries with this novel interlayer demonstrate superiorities in capacity, variable discharge/charge rate, Electrochemical Impedance Spectroscopy (EIS) and Cyclic Voltammetry (CV) characteristics.

\section{Introduction}

The lithium-sulfur ( $\mathrm{Li}-\mathrm{S})$ battery has been explored for decades due to its high theoretical specific energy density $\left(2600 \mathrm{~mW} \mathrm{~h} \mathrm{~g}^{-1}\right)$ and capacity $\left(1675 \mathrm{~mA} \mathrm{~h} \mathrm{~g}^{-1}\right)$, far exceeding those of prevailing Li-ion batteries. ${ }^{1-3}$ These characteristics combined with the wide distribution and environmental friendliness of sulfur enable the Li-S battery to be one of the most promising energy storage devices to satisfy the demands for high capacity and long endurance. Nevertheless, this system is confronted with some challenges. Its practical capacity is lower than the theoretical value due to the poor conductivity of sulfur and the cathode structure is apt to collapse for the volume change of $79.2 \%$ from sulfur to the reduction $\mathrm{Li}_{2} \mathrm{~S}_{2} / \mathrm{Li}_{2} \mathrm{~S}$. Primarily, soluble polysulfide intermediates give rise to "shuttle effect" that leads to sulfur loss, damages cycle stability and reduces coulombic efficiency. ${ }^{4-9}$ Efforts have been extensively devoted to addressing these problems. Carbonaceous materials as sulfur hosts can not only establish conductive framework but also relieve "shuttle effect" by physical absorption of carbon pores. ${ }^{10-14}$ Modified carbon

${ }^{a}$ School of Materials Science and Engineering, Hefei University of Technology, Hefei 230009, China.E-mail: lhqjs@hfut.edu.cn

${ }^{b}$ School of Electronic Science and Applied Physics, Hefei University of Technology, Hefei 230009, China materials by doping element $\mathrm{N}$ or functional group intensify the adsorption of polysulfides by chemical interaction. ${ }^{15-20}$ Conductive polymers are also introduced to mitigate diffusion of polysulfides from the cathode..$^{21-23}$ Although these methods improved the capability of Li-S battery, the inevitable shortcomings lie in complicate fabrication of materials and low sulfur content in cathode.

Recently, the insertion of carbon interlayer between pure sulfur cathode and separator attracts people's attention. Carbon interlayer prepared by convenient manufacture serves as both the upper current collector and barrier of soluble polysulfide intermediates. ${ }^{24-28}$ However, it is noted that physical interaction between nonpolar carbon and polar sulfides is limited. ${ }^{17}$ Therefore, it is necessary to modify carbon interlayer to intensify the inhibition of shuttle effect.

In traditional configuration, polar metal oxides (such as $\mathrm{Al}_{2} \mathrm{O}_{3}, \mathrm{TiO}_{2}, \mathrm{MnO}_{2}$, and $\mathrm{SnO}_{2}$ ) have been added into $\mathrm{S} / \mathrm{C}$ cathode to improve the chemical adsorption of polysulfides by strong electrostatic attraction of metal-oxygen bond. ${ }^{29-32}$ Inspired by the idea, we applied the technique of electron beam evaporation (EBE) to deposit $\mathrm{Al}_{2} \mathrm{O}_{3}$ on carbon paper made by carbonizing commercial filter paper. Fig. 1 is the schematic illustrations of manufacturing material and assembling battery. Electron beam evaporation as a method of physical vapor deposition can produce nano-scale material coating of pure phase on the 


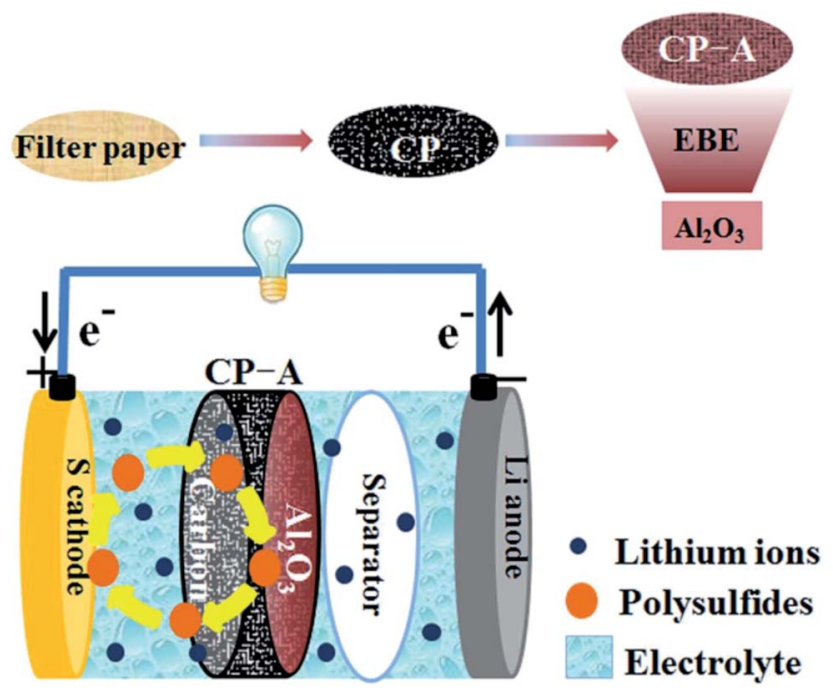

Fig. 1 Schematic illustrations of preparing $\mathrm{Al}_{2} \mathrm{O}_{3}$-deposited carbon paper and the configuration of the Li-S battery with CP-A interlayer.

substrate with benign adhesivity at a high deposition speed. This technique has been applied in Li-ion battery to obtain great improvement of performance. ${ }^{33}$ To the best of our knowledge, it has not been reported to be applied in Li-S battery. Furthermore, the $\mathrm{Al}_{2} \mathrm{O}_{3}$-deposited dual-faced carbon interlayer is also seldom reported in literatures. The carbon side of the interlayer toward the cathode serves as a conductive reservoir with physical interception of sulfur series. The $\mathrm{Al}_{2} \mathrm{O}_{3}$ side toward the separator serves as a lock gate to obstruct leaky sulfides. Li-S battery with this novel interlayer demonstrates remarkable enhancement of performances compared to that with single carbon interlayer. We systematically investigated the effects of the $\mathrm{Al}_{2} \mathrm{O}_{3}$-deposited interlayer on capabilities of $\mathrm{Li}-\mathrm{S}$ battery.

\section{Experimental section}

\subsection{Preparation of sulphur cathode}

$70 \mathrm{wt} \%$ sulfur powder (chemical purity, Sinopharm Chemical Reagent Co., Ltd.) and $20 \mathrm{wt} \%$ acetylene black (purity 99.9\%, Shanxi Luan Group Co., Ltd.) were ground uniformly in an agate mortar, then directly mixed with $10 \mathrm{wt} \%$ polyvinylidene fluoride (PVDF) (Arkema HSV900, France) in $N$-methyl-2-pyrrolidinone (NMP) (purity 99.9\%, Sinopharm Chemical Reagent Co., Ltd.) to form homogeneous slurry. The slurry was casted on $\mathrm{Al}$ foil (purity 99.8\%, $20 \mu \mathrm{m}$ thick) and dried at $50{ }^{\circ} \mathrm{C}$ for 24 hours. Next, it was punched into disks of $12 \mathrm{~mm}$ in diameter as sulfur cathodes. The sulfur loading is $0.70 .8 \mathrm{mg} \mathrm{cm}^{-2}$.

\subsection{Preparation of interlayer}

A free-standing carbon paper was prepared by calcining commercial filter paper (Hangzhou Special Paper Co., Ltd.) at $1000{ }^{\circ} \mathrm{C}$ for 2 hours under argon atmosphere in a tube furnace, with a heating rate of $3{ }^{\circ} \mathrm{C} \mathrm{min}^{-1}$ and a flow rate of $30 \mathrm{~mL} \mathrm{~min}^{-1}$. When cooled down to ambient temperature, prepared carbon paper with thickness of about $80 \mu \mathrm{m}$ (measured by a vernier gauge) was directly placed in an electronbeam evaporator (DZS-500, China) for $\mathrm{Al}_{2} \mathrm{O}_{3}$ deposition. The base pressure of vacuum chamber is $7.0 \times 10^{-4} \mathrm{~Pa}$. Under the current of electron beam of $40 \mathrm{~mA}, \mathrm{Al}_{2} \mathrm{O}_{3}$ target (purity 99.5\%) loaded into a $\mathrm{Cu}$ crucible was pre-evaporated until the rate indicator was $1 \AA^{-1}$. Then the shutter was opened and $\mathrm{Al}_{2} \mathrm{O}_{3}$ was evaporated for 200 seconds on one face of the carbon paper substrate. The carbon papers with and without $\mathrm{Al}_{2} \mathrm{O}_{3}$ deposition are named after CP-A and CP, respectively. Both were cut into circle slices of $12 \mathrm{~mm}$ in diameter as interlayers.

\subsection{Material characterization}

The morphologies and element mappings of the synthesized material were observed using field-emission scanning electron microscopy (FESEM) on SU8020 (Hitachi, Japan) combined with energy dispersive X-ray spectroscopy (EDS). X-ray photoelectron spectroscopy (XPS) measurement was performed on a Thermo ESCALAB 250 energy spectrometer (USA) with a monochromatic Al $K_{\alpha}(1486.60 \mathrm{eV})$ X-ray source. All binding energies were labeled taking $\mathrm{C} 1 \mathrm{~s}$ at $284.8 \mathrm{eV}$ as a reference according to the testing environment. Current-Voltage $(I-V)$ tests of interlayers were carried out on a Keithley 4200 semiconductor characterization system (USA) to compare the resistances of carbon paper before and after $\mathrm{Al}_{2} \mathrm{O}_{3}$ deposition.

\subsection{Electrochemical measurements}

Three kinds of CR2032-type coin cells were assembled with bare $\mathrm{S}$ cathode, the S cathode with $\mathrm{CP}$ and with $\mathrm{CP}-\mathrm{A}$ interlayer, respectively. Lithium foil (purity 99.95\%, $0.6 \mathrm{~mm}$ thick and $15.8 \mathrm{~mm}$ in diameter) was used as anodic electrode and Celgard 2400 as separator. The electrolyte purchased from Suzhou Qianmin Chemical Reagent Co., Ltd. was $1 \mathrm{M}$ bis(trifluoromethane) sulfonamide lithium salt (LITFSI) dissolved in a mixed solution of 1,3-dioxolane (DOL) and dimethyl ether (DME) $(1: 1, \mathrm{v} / \mathrm{v})$ with $1 \mathrm{wt} \% \mathrm{LiNO}_{3}$ added.

The cells were assembled in an argon-filled glove box (Etelux Lab 2000, China) with oxygen and water contents below $0.1 \mathrm{ppm}$. Galvanostatic cycling tests were conducted between 1.5 and $3.0 \mathrm{~V}$ on a Neware BTS2300 system (Shenzhen, China) at room temperature. Cyclic Voltammetry (CV) measurements were performed at a scan rate of $0.2 \mathrm{mV} \mathrm{s}^{-1}$ and electrochemical impedance spectra (EIS) measurements were conducted from $100 \mathrm{kHz}$ to $0.1 \mathrm{~Hz}$ with potentiostatic amplitude of $5 \mathrm{mV}$ on a CHI 760D (Shanghai, China) electrochemical workstation.

\section{Results and discussion}

The carbon paper has a netlike structure of weaved and stacked fibers as shown in Fig. 2(a). And the magnification image demonstrates there is a hierarchical pore size distribution in fibers, which contributes to the accommodation of sulfur series, ion transfer and infiltration of electrolyte. ${ }^{10}$ After depositing $\mathrm{Al}_{2} \mathrm{O}_{3}$, no apparent particle or bulk can be observed form the SEM image of Fig. 2(b). However, $\mathrm{Al}_{2} \mathrm{O}_{3}$ side of the carbon paper appears to be darker compared with the carbon side without deposition, as shown in the inset. In addition, distributions of 

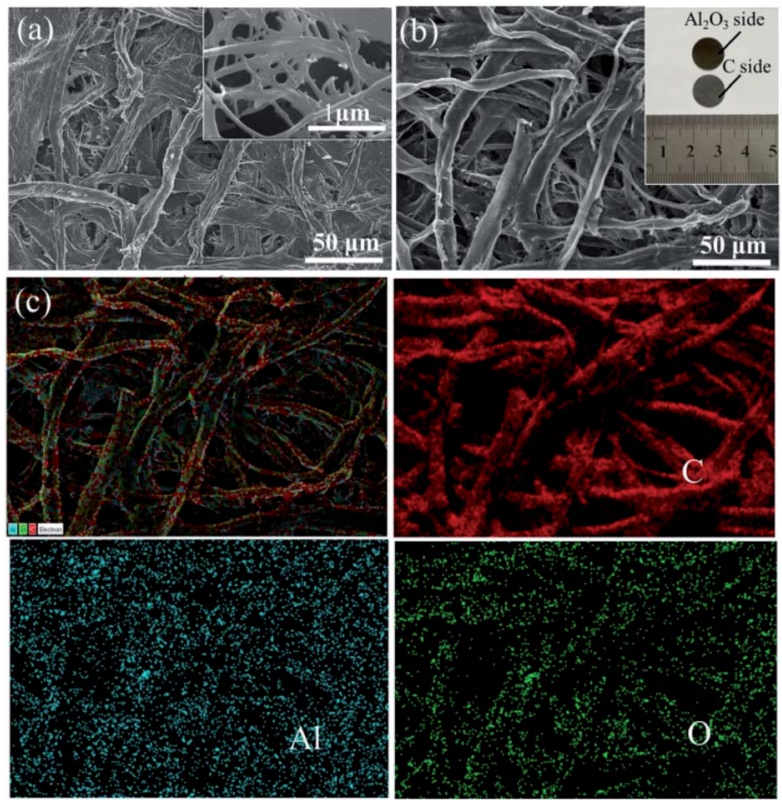

Fig. 2 FESEM morphologies of (a) CP (inset: a magnification area) and (b) CP-A with a digital photograph inset of the $\mathrm{Al}_{2} \mathrm{O}_{3}$ side and carbon side; (c) overlap of element mappings and separate element mappings for $\mathrm{C}, \mathrm{Al}$ and $\mathrm{O}$ corresponding to CP-A shown in (b).

element aluminum and oxygen shown in Fig. 2(c) confirm one side of the carbon paper has been homogeneously coated by $\mathrm{Al}_{2} \mathrm{O}_{3}$ referenced to the distribution of carbon. The chemical state of the deposition was further investigated by XPS analysis. The peak at $74.3 \mathrm{eV}$ of $\mathrm{Al} 2$ p spectrum shown in Fig. 3(a) and the peak at 531.5 eV of $\mathrm{O} 1 \mathrm{~s}$ spectrum shown in Fig. 3(b) correspond to the binding energies of $\mathrm{Al}^{3+}$ and $\mathrm{O}^{2-}$ in $\mathrm{Al}_{2} \mathrm{O}_{3}$, respectively and no metallic $\mathrm{Al}$ or other valence state of $\mathrm{Al}$ exists, which agrees well with the element mappings. ${ }^{34}$

The resistances of CP and CP-A were measured by $I-V$ tests as shown in Fig. 4. The samples were cut into the same size of squares with $1 \mathrm{~cm}$ in length, and silver glue was painted on two opposite sides of each square. $I-V$ results were obtained by probes contacting on the two sides with silver glue. Both straight lines show reversible ohm characteristics in a sweep voltage range of -0.01 to $+0.01 \mathrm{~V}$, which means deposition of $\mathrm{Al}_{2} \mathrm{O}_{3}$ did not induce extra contact potential barrier that is socalled Schottky effect, and thereby did not change the transport property of electron. By calculating the slopes of straight lines, the ohm resistances of CP and CP-A are separately 9.3 and $12.5 \mathrm{ohm}$, which indicates the conductivity of carbon paper reduces after being coated by insulating $\mathrm{Al}_{2} \mathrm{O}_{3}$. Therefore, the conductive carbon side of interlayer is supposed to face toward the cathode with high sulfur loading, resulting into good conductive interface to activate sulfur as upper current collector. During discharge, soluble intermediates first encounter the intercept of physical barrier and further chemical adsorption from polar $\mathrm{Al}_{2} \mathrm{O}_{3}$ facing toward the separator.

The electrochemical performances of the bare $\mathrm{S}$ cathode, $\mathrm{S}$ cathode with CP interlayer and S cathode with CP-A interlayer for Li-S batteries were investigated. In Fig. 5(a), the Li-S battery
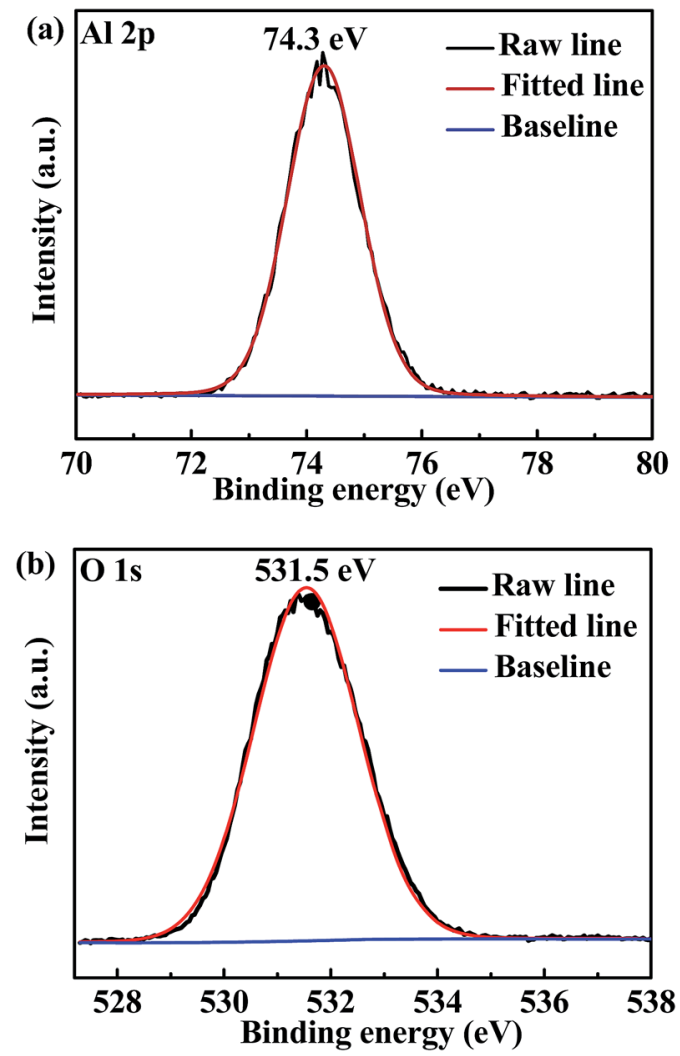

Fig. 3 (a) Al 2p and (b) $\bigcirc$ 1s XPS spectra of CP-A.

with CP-A interlayer presents the initial discharge capacity of $1352 \mathrm{~mA} \mathrm{~h} \mathrm{~g}^{-1}$ at $0.2 \mathrm{C}\left(1 \mathrm{C}=1675 \mathrm{~mA} \mathrm{~g}^{-1}\right)$ approaching to $81 \%$ utilization of sulfur, higher than $1198 \mathrm{~mA} \mathrm{~h} \mathrm{~g}^{-1}$ of the battery with CP interlayer. Although the faces to cathodes are both conductive carbon in these two batteries, the extra deposited $\mathrm{Al}_{2} \mathrm{O}_{3}$ in CP-A mitigates the escape of soluble polysulfides from interlayer during discharge, and these reduction products intercepted into interlayer are reactivated. In result, the battery with CP-A interlayer delivers higher initial capacity. Over 60 cycles the battery with CP-A interlayer retains the highest reversible capacity

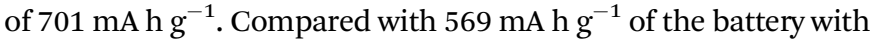
CP interlayer, it can be deduced that $\mathrm{Al}_{2} \mathrm{O}_{3}$ effectively alleviates the decay of capacity. On the other hand, the poor initial capacity (711 $\mathrm{mA} \mathrm{h}^{-1}$ ) and 60th capacity (329 $\mathrm{mA} \mathrm{h}^{-1}$ ) of the bare sulfur electrode shows inferior sulfur utilization and capacity retention.

Fig. 5(b) is the 1st and 30th discharge/charge profiles of three samples at $0.2 \mathrm{C}$, which all exhibit two typical discharge plateaus of $\mathrm{Li}-\mathrm{S}$ batteries. The higher plateau at about 2.3-2.2 V corresponds to the transformation from sulfur to soluble $\operatorname{Li}_{2} \mathrm{~S}_{x}(4 \leq x$ $\leq 8$ ), and the lower at about 2.1-2.0 V corresponds to the formation of insoluble $\mathrm{Li}_{2} \mathrm{~S}_{2} / \mathrm{Li}_{2} \mathrm{~S} \cdot{ }^{35,36}$ It is noticed that for the bare $S$ cathode and the $S$ cathode with $\mathrm{CP}$ interlayer, the voltage gaps between the second discharge and charge plateaus gradually increase as discharge/charge cycles increase. On the contrary, no evident change is detected in the $\mathrm{S}$ cathode with CP-A interlayer. Moreover, the voltage gap of the $S$ cathode with CP-A interlayer is always the narrowest, whether in the 1st or 30th cycle. It is attributed to the adsorption of polysulfides by 


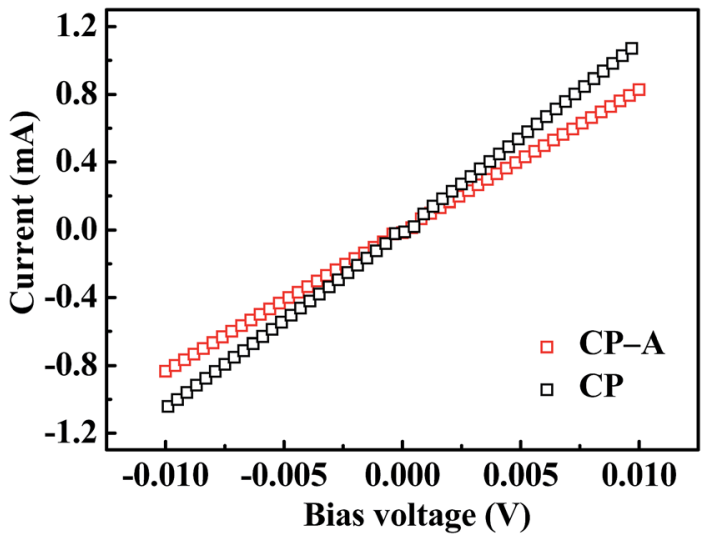

Fig. 4 Current-voltage profiles of CP and CP-A.

$\mathrm{Al}_{2} \mathrm{O}_{3}$ that inhibits the shuttle effect and reduces the polarization of Li-S battery. Besides, the low-voltage plateaus of $\mathrm{S}$ cathode with CP-A interlayer are both longer than the others, suggesting more soluble polysulfides intercepted to participate in reduction reaction and produce more discharge capacity.

To further investigate the effect of CP-A interlayer, discharge/ charge cycles at $0.5 \mathrm{C}$ and variable rate were tested. At $0.5 \mathrm{C}$ as shown in Fig. 5(c), the batteries with bare sulfur cathode, CP-A interlayer and $\mathrm{CP}$ interlayer deliver initial capacities of $588 \mathrm{~mA} \mathrm{~h} \mathrm{~g}^{-1}, 1253 \mathrm{~mA} \mathrm{~h} \mathrm{~g}^{-1}$ and $1117 \mathrm{~mA} \mathrm{~h} \mathrm{~g}^{-1}$, respectively. After 85 cycles, the specific capacities of the bare sulfur cathode and the cathode with CP separately reduce to $441 \mathrm{~mA} \mathrm{~h} \mathrm{~g}^{-1}$ and $361 \mathrm{~mA} \mathrm{~h} \mathrm{~g}{ }^{-1}$, while the cathode with CP-A delivers desirable capacity of $700 \mathrm{~mA} \mathrm{~h} \mathrm{~g}{ }^{-1}$ even after 120 cycles, presenting superior capability and extended cycling life. Furthermore, the cathode with CP drops in capacity by more than $50 \%$ after 85 cycles, while the cathode with CP-A drops in capacity by less than $50 \%$ after 120 cycles. For the bare S cathode, although the dropping ratio of capacity is close to that for the cathode with CP-A, it can be observed that the capacity obtained from the S cathode is less than $50 \%$ of that observed from the cathode with CP-A. This is probably because that on account of bad conductivity of the S cathode, only a small amount of active material could participate in reaction and accordingly the small amount of soluble polysulfides could be effectively blocked only by conventional separator. However, when the conductive interlayer was inserted, the capacity of the sulfur cathode was largely released. In result, the dropping ratio of capacity of the cathode with $\mathrm{CP}$ is even higher than that for the S cathode. But the cathode with CPA has both benign sulfur utilization and capacity retaining. As for coulombic efficiency, the introduction of $\mathrm{LiNO}_{3}$ in electrolyte makes all batteries more than $95 \%$. Fig. 5 (d) is the variable rate test from $0.1 \mathrm{C}$ to $1 \mathrm{C}$. Capacities of batteries gradually decay ascribed to gradually severe polarization as rate increases. Even so, the cathode with CP-A interlayer delivers the highest capacity in every rate stage and when rate returns to $0.1 \mathrm{C}$, it can also regain the majority of capacity, presenting the highest discharge capacity among the samples. Therefore, the cathode with CP-A interlayer not only improves the cycle capability, but also exhibits very good reversible behaviour.
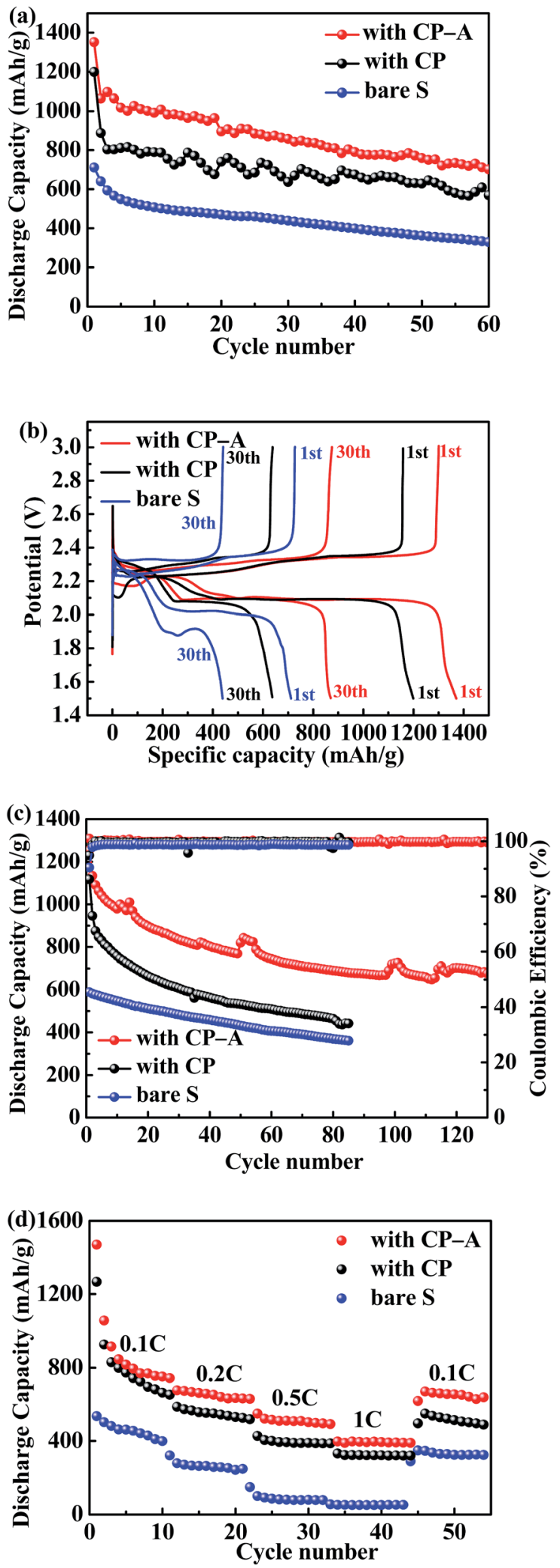

Fig. 5 (a) Cycling performances at $0.2 C$, (b) discharge/charge profiles of the initial and 30th cycle at $0.2 \mathrm{C}$, (c) cycling performances at $0.5 \mathrm{C}$ and (d) rate capabilities of the bare $\mathrm{S}$ cathode, $\mathrm{S}$ cathode with $\mathrm{CP}$ interlayer and S cathode with CP-A interlayer. 


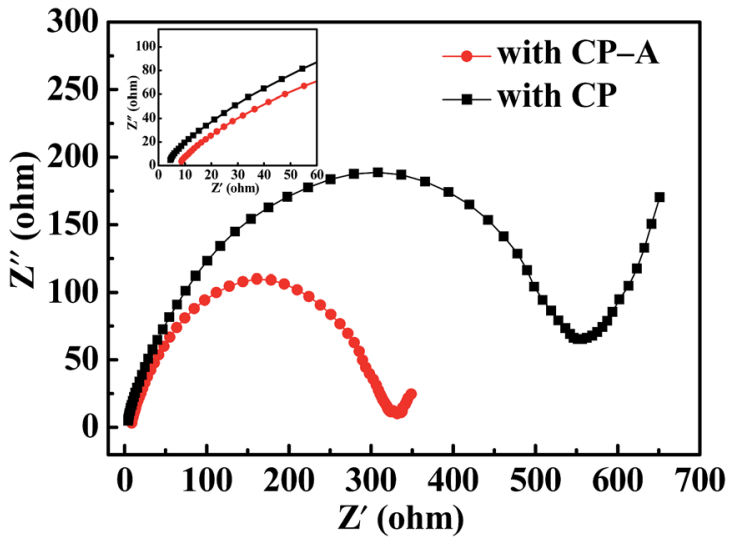

Fig. 6 EIS curves of the batteries with CP and CP-A interlayers and the inset of enlarge curves at a small scale.

EIS tests of the S cathode with CP interlayer and S cathode CP-A interlayer were performed before cycling, respectively. Both curves in Fig. 6 display the analogy profiles with a semicircle in high frequency corresponding to charge transfer resistance and an inclined line in low frequency corresponding to ion diffusion resistance. Differently, for the cathode with CP-A, the left point of intersection of the semicircles and abscissa axis shifts right a little compared with the cathode with $\mathrm{CP}$, which denotes it has a little higher internal resistance which originates from the ohm resistances of electrolyte, electrode materials, current collector and contact interface. ${ }^{37}$ This is because non-conductive $\mathrm{Al}_{2} \mathrm{O}_{3}$ increases the ohm resistance of the electrode material in accordance with the analysis of $I-V$ test. Nevertheless, the much smaller diameter of the semicircle signifies the battery with CP-A interlayer possesses a much lower charge transfer resistance than that with $\mathrm{CP}$ interlayer. This is possibly because deposited $\mathrm{Al}_{2} \mathrm{O}_{3}$ particles not only have adsorption of polysulfides but also increase the contact sites with electrolyte leading to more electrolyte uptake. The synergetic effects facilitate the charge transfer. The result supports the superior cycling capability of the battery with CP-A interlayer.

Fig. 7 shows the $\mathrm{CV}$ profiles of the batteries with $\mathrm{CP}$ and with CP-A interlayer, respectively with typical CV shapes of conventional Li-S batteries. It is noteworthy that the initial CV cycles of both batteries obviously deviate from the subsequent two cycles, which is ascribed to the activation of batteries and redistribution of sulfur on favorable sites. ${ }^{38}$ Besides, one broad oxidation peak in the initial scan evolves into two adjacent peaks at around $2.45-2.48 \mathrm{~V}$ and $2.47-2.52 \mathrm{~V}$ in the next scans, also indicating electrochemical activation has taken place. ${ }^{39}$ These two oxidation peaks correspond to the oxidation of $\mathrm{Li}_{2} \mathrm{~S}_{2} / \mathrm{Li}_{2} \mathrm{~S}$ to polysulfides and then to sulfur, and the reduction peaks at about 1.96-1.98 $\mathrm{V}$ and $2.26-2.27 \mathrm{~V}$ correspond to the reduction of sulfur to $\mathrm{Li}_{2} \mathrm{~S}_{x}(4 \leq x \leq 8)$ and then to $\mathrm{Li}_{2} \mathrm{~S}_{2} / \mathrm{Li}_{2} \mathrm{~S} .{ }^{40}$ Differently, the CV profiles of the battery with CP-A interlayer almost overlap after the initial cycle and the peak intensity has no obvious change as shown in Fig. 7(b), suggesting benign electrochemical reversibility and slow capacity fading. Contrarily, the
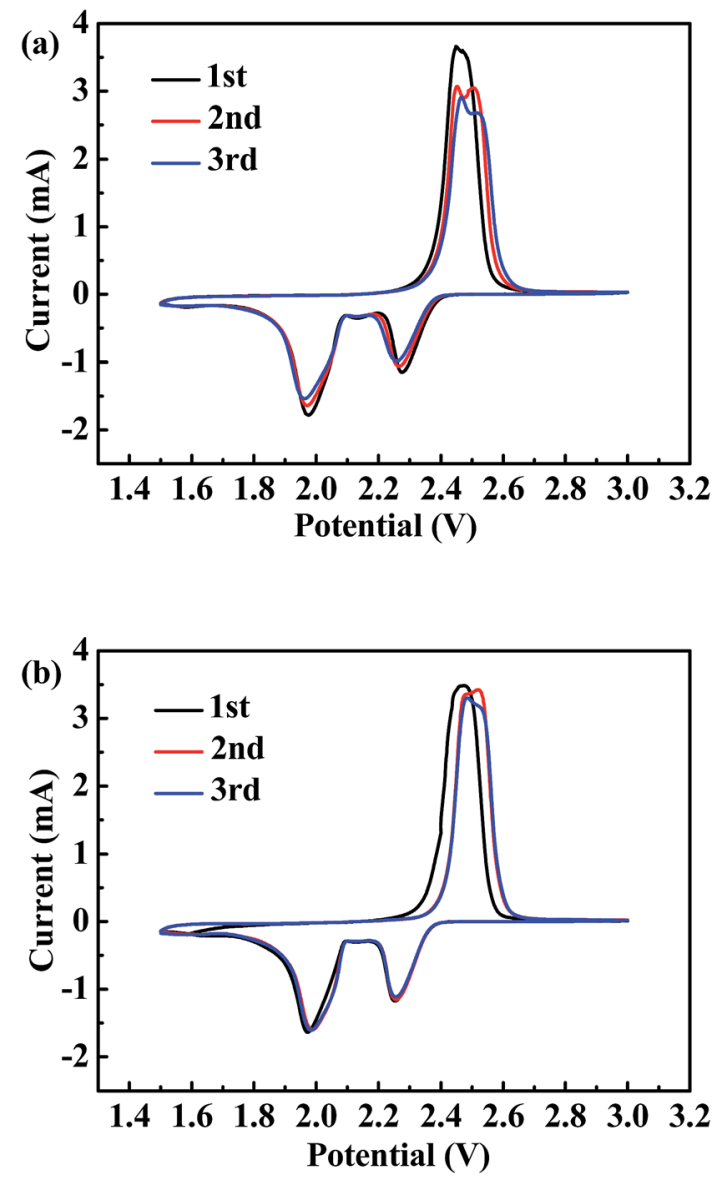

Fig. 7 CV curves of (a) the battery with $\mathrm{CP}$ and (b) the battery with $\mathrm{CP}$ A interlayer.

profiles of the battery with CP interlayer shown in Fig. 7(a) in the following two cycles change evidently and peak intensities decrease distinctly. Furthermore, in terms of the peak positions, the reduction peaks shift lower and the oxidation peaks shift higher in the battery with CP interlayer, indicating continually enlarged polarization during redox. While no shift is detected in
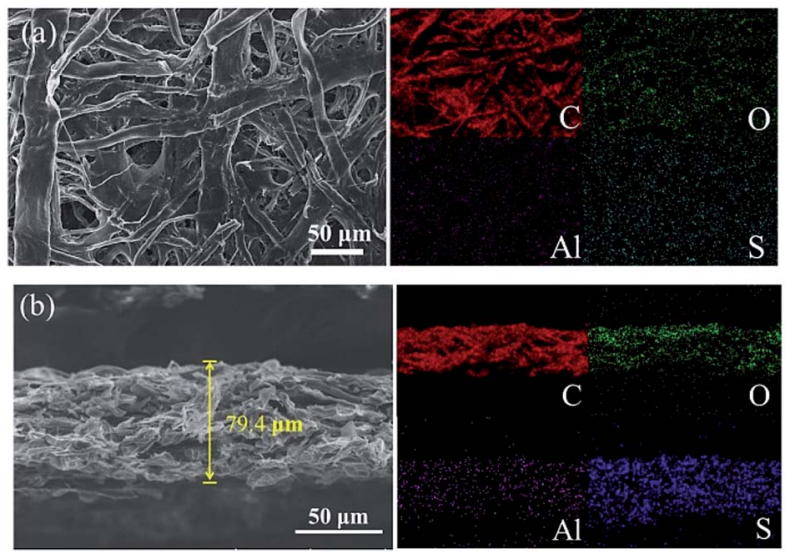

Fig. 8 FESEM images of (a) the face and (b) cross sections with their corresponding element mappings for $\mathrm{CP}-\mathrm{A}$ interlayer after cycling. 


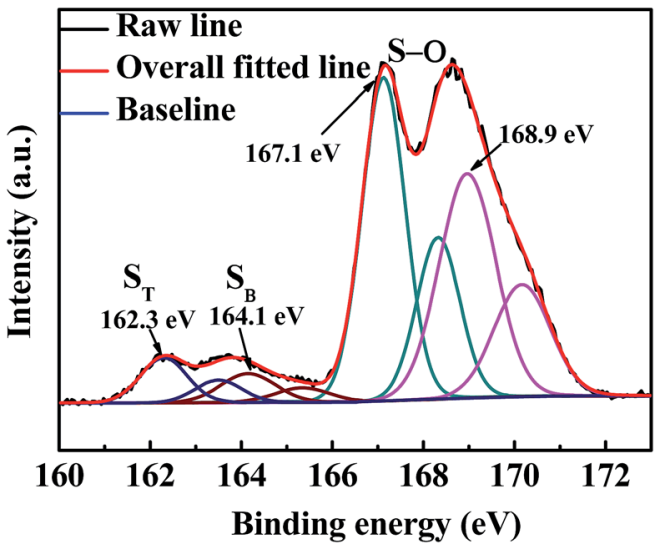

Fig. 9 S 2p XPS spectra for the CP-A interlayer after 100 cycles.

the battery with CP-A interlayer, indicating the electrochemical state is stable and polarization has not been deteriorated. The observations of CV profiles agree well with the electrochemical performances of the two kinds of batteries.

We disassembled a coin cell at the state of full charge after 100 discharge/charge cycles and washed the CP-A interlayer with 1,3-dioxolane solvent in a glove box for ex situ characterization. The FESEM images of the face and cross sections with corresponding element mappings are shown in Fig. 8. The interlayer morphology of a netlike structure was retained and no apparent fracture of fibers is observed from Fig. 8(a). What's more, compared to the initial thickness of about $80 \mu \mathrm{m}$ of carbon paper, the thickness of cross section almost has no change as shown in Fig. 8(b). That means the interlayer has prominent mechanical strength to endure the volume change during discharge/charge reaction. $\mathrm{Al}_{2} \mathrm{O}_{3}$ as a kind of ceramic material is conducive to enhancing the rigidity of carbon paper to keep integrity of carbon paper even after 100 cycles. Some dark areas can be observed in the interlaced structure of carbon paper as shown in Fig. 8(b) FESEM picture. Because the carbon paper has plenty of pores and holes, and the escaped polysulfides could not fill up all the interspaces entirely and they may adhere to the surfaces of carbon fibers or fill in more small pores so that relative large voids are visible from the picture. Additionally, although no bulk sulfur-series particles can be observed from the image, the EDS mappings of element C, O, Al and $\mathrm{S}$ indicate sulfur series are effectively intercepted into the interlayer. This also can be confirmed by ex situ high-resolution $\mathrm{S} 2 \mathrm{p}$ XP spectrum of the cycled CP-A interlayer shown in Fig. 9. It is noted that only the $2 \mathrm{p}_{3 / 2}$ component of the $2 \mathrm{p}_{3 / 2} / 2 \mathrm{p}_{1 / 2}$ doublet is labeled as convention. Each $\mathrm{S} 2 \mathrm{p}_{3 / 2} / 2 \mathrm{p}_{1 / 2}$ doublet was fitted according to the principle that the peak area ratio is $2: 1$, with $1.2 \mathrm{eV}$ of splitting energy and same full width at half maximum. The binding energy located at 167.1 and $168.9 \mathrm{eV}$ are attributed to S-O bonding with characteristics of sulphite and sulphate, which originate from residual bis(trifluoromethane) sulfonamide lithium salt (LITFSI) and exposure to air outside glove box before test, respectively. ${ }^{41}$ The binding energy of $164.1 \mathrm{eV}$ is assigned to bridging $\mathrm{S}\left(\mathrm{S}_{\mathrm{B}}\right)$ originating from element sulfur. The binding energy of $162.3 \mathrm{eV}$ is assigned to terminal $\mathrm{S}\left(\mathrm{S}_{\mathrm{T}}\right)$

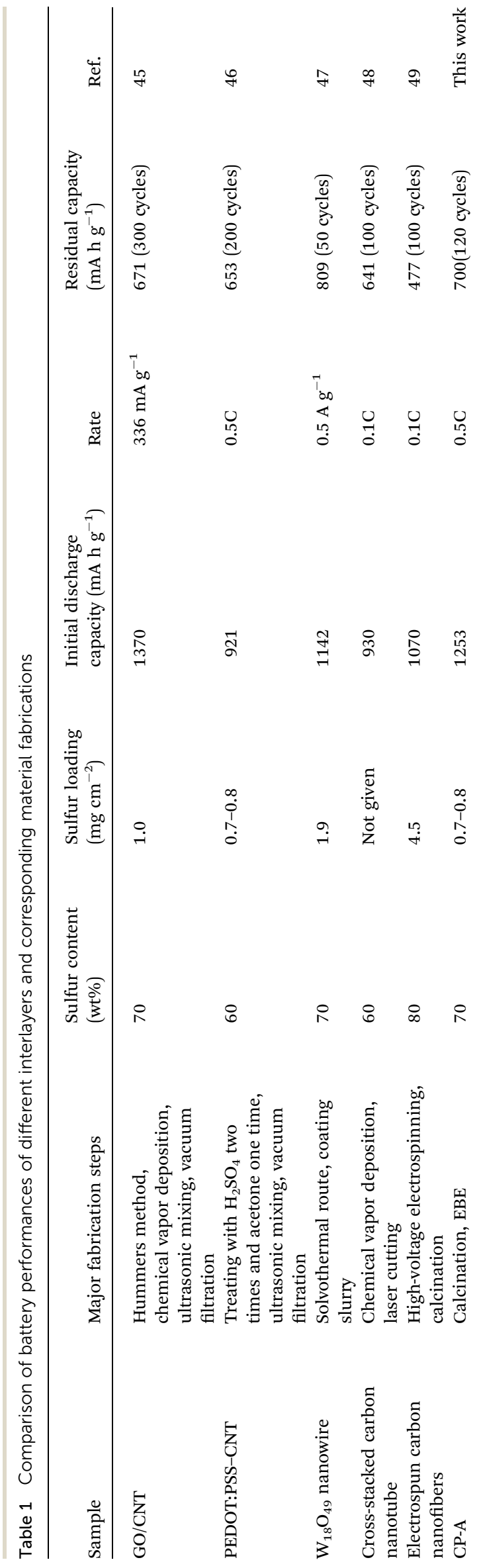


originating from polysulfide compounds, indicating reduction intermediates deviating from cathode are hold up and retained into CP-A interlayer for reutilization. ${ }^{\mathbf{4 2 - 4 4}}$ These ex situ characterizations are consistent with previous electrochemical analyses.

Table 1 is a comparison with other reported interlayers in recent years. It indicates the Li-S battery with the CP-A interlayer presents comparable electrochemical performances by uncomplicated fabrication.

\section{Conclusions}

$\mathrm{Al}_{2} \mathrm{O}_{3}$-deposited carbon paper by the EBE technique as interlayer is inserted into the traditional Li-S battery.

Compared with the sulfur cathode with $\mathrm{CP}$ interlayer, the electrochemical performances of that with CP-A interlayer are notably enhanced. We conclude that firstly, the $\mathrm{Al}_{2} \mathrm{O}_{3}$ coating raises the mechanical strength of carbon paper, enables physical morphology of the interlayer intact to undertake large volume change during discharge/charge cycles. Secondly, the two-faced interlayer ensures good conductivity of interface between cathode and the carbon side. Lastly, chemical adsorption of polar sulfur species by polar $\mathrm{Al}_{2} \mathrm{O}_{3}$ has stronger inhibition of shuttle effect than physical absorption of nonpolar porous carbon. Besides, extra contact sites brought by deposition may raise the uptake of electrolyte to facilitate electrochemical reaction. In all, this method of physical vapor deposition provides an effectual avenue for manufacturing material applied in Li-S batteries.

\section{Conflicts of interest}

There are no conflicts to declare.

\section{Acknowledgements}

The authors gratefully acknowledge the support of the "Strategic Priority Research Program" of the Chinese Academy of Science (Grant No. XDA03040000).

\section{References}

1 P. G. Bruce, S. A. Freunberger, L. J. Hardwick, et al., Nat. Mater., 2012, 11, 19-29.

2 J. Wang, Y. Wu, Z. Shi, et al., Electrochim. Acta, 2014, 144, 307-314.

3 B. Zhang, X. Qin, G. R. Li, et al., Energy Environ. Sci., 2010, 3, 1531-1537.

4 A. Manthiram, Y. Fu and Y. S. Su, Acc. Chem. Res., 2012, 46, 1125-1134.

5 A. Manthiram, Y. Fu, S. H. Chung, et al., Chem. Rev., 2014, 114, 11751-11787.

6 S. S. Zhang, J. Power Sources, 2013, 231, 153-162.

7 S. Evers, T. Yim and L. F. Nazar, J. Phys. Chem. C, 2012, 116, 19653-19658.

8 Y. Yang, G. Zheng and Y. Cui, Chem. Soc. Rev., 2013, 42, 30183032.
9 X. Ji and L. F. Nazar, J. Mater. Chem., 2010, 20, 9821-9826.

10 H. Ye, Y. X. Yin, S. Xin, et al., J. Mater. Chem. A, 2013, 1, 66026608.

11 R. Sahore, L. P. Estevez, A. Ramanujapuram, et al., J. Power Sources, 2015, 297, 188-194.

12 J. T. Lee, Y. Zhao, H. Kim, et al., J. Power Sources, 2014, 248, 752-761.

13 Y. Zhang, Y. Zhao, A. Konarov, et al., J. Alloys Compd., 2015, 619, 298-302.

14 M. Depardieu, R. Janot, C. Sanchez, et al., RSC Adv., 2014, 4, 23971-23976.

15 S. Wang, Z. Zhao, H. Xu, et al., Electrochim. Acta, 2015, 173, 282-289.

16 Y. Qu, Z. Zhang, X. Zhang, et al., Carbon, 2015, 84, 399-408.

17 J. Song, T. Xu, M. L. Gordin, et al., Adv. Funct. Mater., 2014, 24, 1243-1250.

18 J. Qu, S. Lv, X. Peng, et al., J. Alloys Compd., 2016, 671, 17-23. 19 L. Ji, M. Rao, H. Zheng, et al., J. Am. Chem. Soc., 2011, 133, 18522-18525.

20 L. Zhang, L. Ji, P. A. Glans, et al., Phys. Chem. Chem. Phys., 2012, 14, 13670-13675.

21 J. Wang, J. Yang, J. Xie, et al., Adv. Mater., 2002, 14, 963-965. 22 W. Zhou, Y. Yu, H. Chen, et al., J. Am. Chem. Soc., 2013, 135, 16736-16743.

23 Y. Zhang, Y. Zhao, A. Yermukhambetova, et al., J. Mater. Chem. A, 2013, 1, 295-301.

24 Y. S. Su and A. Manthiram, Chem. Commun., 2012, 48, 88178819.

25 C. Zu, Y. S. Su, Y. Fu, et al., Phys. Chem. Chem. Phys., 2013, 15, 2291-2297.

26 T. G. Jeong, Y. H. Moon, H. H. Chun, et al., Chem. Commun., 2013, 49, 11107-11109.

27 H. Deng, L. Yao, Q. A. Huang, et al., Mater. Res. Bull., 2016, 84, 218-224.

28 J. Yang, F. Chen, C. Li, et al., J. Mater. Chem. A, 2016, 4, 14324-14333.

29 X. Liu, Z. Shan, K. Zhu, et al., J. Power Sources, 2015, 274, 8593.

30 Z. W. Seh, W. Li, J. J. Cha, et al., Nat. Commun., 2013, 4, 1331.

31 X. Liang, C. Hart, Q. Pang, et al., Nat. Commun., 2015, 6, 5682.

32 L. P. Zhang, Y. F. Wang, S. Q. Gou, et al., J. Phys. Chem. C, 2015, 119, 28721-28727.

33 R. Z. Hu, M. Q. Zeng and M. Zhu, Electrochim. Acta, 2009, 54, 2843-2850.

34 D. Leinen, G. Lassaletta, A. Fernández, et al., J. Vac. Sci. Technol., A, 1996, 14, 2842-2848.

35 Y. Yang, G. Zheng and Y. Cui, Chem. Soc. Rev., 2013, 42(7), 3018-3032.

36 S. S. Zhang, J. Power Sources, 2013, 231, 153-162.

37 X. Zhou, J. Xie, J. Yang, et al., J. Power Sources, 2013, 243, 9931000.

38 Y. Yang, W. Sun, J. Zhang, et al., Electrochim. Acta, 2016, 209, 691-699.

39 S. Xin, Y. You, H. Q. Li, et al., ACS Appl. Mater. Interfaces, 2016, 8(49), 33704-33711. 
40 R. Elazari, G. Salitra, A. Garsuch, et al., Adv. Mater., 2011, 23, 5641-5644.

41 Q. Pang, D. Kundu, M. Cuisinier, et al., Nat. Commun., 2014, 5, 4759.

42 D. Brion, Appl. Surf. Sci., 1980, 5(2), 133-152.

43 J. Balach, T. Jaumann, M. Klose, et al., Adv. Funct. Mater., 2015, 25(33), 5285-5291.

44 J. Liu, L. Yuan, K. Yuan, et al., Nanoscale, 2016, 8(28), 1363813645.
45 J. Q. Huang, Z. L. Xu, S. Abouali, et al., Carbon, 2016, 99, 624632.

46 A. Wang, G. Xu, B. Ding, et al., ChemElectroChem, 2017, 4, 362-368.

47 W. Zhang, C. Lin, S. Cong, et al., RSC Adv., 2016, 6, 1523415239.

48 L. Sun, W. Kong, M. Li, et al., Nanotechnology, 2016, 27, 075401.

49 T. Gao, T. H. Le, Y. Yang, et al., Materials, 2017, 10, 376. 